\title{
Impact of Active-Sterile Neutrino Mixing on Supernova Explosion and Nucleosynthesis
}

\author{
Meng-Ru Wu, ${ }^{1}$ Tobias Fischer ${ }^{2}$ Lutz Huther, ${ }^{1}$ Gabriel Martínez-Pinedo, ${ }^{1,3}$ and Yong-Zhong Qian ${ }^{4}$ \\ ${ }^{1}$ Institut für Kernphysik (Theoriezentrum), Technische Universität Darmstadt, \\ Schlossgartenstraße 2, 64289 Darmstadt, Germany \\ ${ }^{2}$ Institute for Theoretical Physics, University of Wroctaw, pl. M. Borna 9, 50-204 Wroctaw, Poland \\ ${ }^{3}$ GSI Helmholtzzentrum für Schwerioneneforschung, Planckstraße 1, 64291 Darmstadt, Germany \\ ${ }^{4}$ School of Physics and Astronomy, University of Minnesota, Minneapolis, MN 55455, USA
}

(Dated: January 6, 2018)

\begin{abstract}
We show that for the active-sterile flavor mixing parameters suggested by the reactor neutrino anomaly, substantial $\nu_{e}-\nu_{s}$ and $\bar{\nu}_{e}-\bar{\nu}_{s}$ conversion occurs in regions with electron fraction of $\approx 1 / 3$ near the core of an $8.8 M_{\odot}$ electron-capture supernova. Compared to the case without such conversion, the neutron-richness of the ejected material is enhanced to allow production of elements from Sr, Y, and $\mathrm{Zr}$ up to $\mathrm{Cd}$ in broad agreement with observations of the metal-poor star HD 122563. Active-sterile flavor conversion also strongly suppresses neutrino heating at times when it is important for the revival of the shock. Our results suggest that simulations of supernova explosion and nucleosynthesis may be used to constrain active-sterile mixing parameters in combination with neutrino experiments and cosmological considerations.

PACS numbers: 14.60.Pq, 97.60.Bw, 26.30.-k
\end{abstract}

The number of active neutrino flavors that participate in weak interactions in the Standard Model has been precisely determined from the $Z^{0}$ decay width to be $2.984 \pm 0.008$ [1]. Nearly all the vacuum mixing parameters for active neutrinos have been measured, except for the mass hierarchy and the $\mathrm{CP}$ violating phase [2]. However, recent results on oscillations of neutrinos from reactors, radioactive sources, and accelerators indicate that the standard mixing scenario involving only the three active flavors may not be complete [3 -7$]$. Light sterile neutrinos $\left(\nu_{s}\right)$ of the $\mathrm{eV}$ mass scale that mix with active neutrinos have been proposed as the simplest extension beyond the standard scenario to explain these anomalies (see [8] for a recent review). The effective relativistic degree of freedom inferred from both Cosmic Microwave Background data and Big Bang Nucleosynthesis studies has large enough uncertainties to also allow such sterile neutrinos to exist [9 11]. There is some tension between the sterile neutrino parameters obtained from different oscillation experiments and cosmological constraints 12 14. This will be resolved by future experiments including solar neutrino measurements [8].

In addition to important implications for experiments and cosmology, mixing of sterile with active neutrinos may lead to multiple Mikheyev-Smirnov-Wolfenstein (MSW) resonances in matter 15 17], which could have interesting effects in supernovae 17 28. In particular, 18] pointed out that for light sterile neutrinos of 1$100 \mathrm{eV}, \nu_{e}-\nu_{s}$ and $\bar{\nu}_{e}-\bar{\nu}_{s}$ conversion could have significant impact on supernova explosion and nucleosynthesis. As such conversion changes the electron fraction $Y_{e}$, and hence the matter potential determining the MSW resonances, this feedback should be included in a full treatment of the problem. Possible feedback effects were noted in 18 and taken into account in some later works (e.g., [22, 23, 28]), which mostly focused on the outer resonances at baryon densities of $\rho<10^{8} \mathrm{~g} \mathrm{~cm}^{-3}$.

In this Letter we examine active-sterile flavor conversion (ASFC) in the region of the inner resonances (IR) where $\rho \sim 10^{9}-10^{12} \mathrm{~g} \mathrm{~cm}^{-3}$ and evaluate its impact on supernova dynamics and nucleosynthesis. A supernova starts when a massive star undergoes gravitational core collapse at the end of its life. Upon reaching supranuclear density, the core bounces to launch a shock and a protoneutron star forms and cools by emitting all three flavors of active (anti)neutrinos. As the shock propagates out of the core, it loses energy by dissociating nuclei in the material falling through it into free nucleons. At the same time, the dissociated material behind the shock gains energy from neutrino heating mainly by the following reactions:

$$
\begin{aligned}
& \nu_{e}+n \rightarrow p+e^{-}, \\
& \bar{\nu}_{e}+p \rightarrow n+e^{+} .
\end{aligned}
$$

This is the essence of the so-called delayed neutrinoheating explosion mechanism [29], which has been shown to result in explosions in recent supernova simulations 30 32. Reactions (1a) and (1b) and their reverse reactions are essential to determining the $Y_{e}$ and hence, nucleosynthesis in any neutrino-heated ejecta [33 35]. ASFC of the $\nu_{e}-\nu_{s}$ and $\bar{\nu}_{e}-\bar{\nu}_{s}$ types may influence the rates of reactions (1a) and (1b), and consequently, affect supernova dynamics and nucleosynthesis.

To treat ASFC in supernovae, we assume effective 2$\nu$ mixing between $\nu_{e}$ and $\nu_{s}\left(\bar{\nu}_{e}\right.$ and $\left.\bar{\nu}_{s}\right)$. This is justified as the magnitude of the mass-squared difference $\delta m^{2} \sim \mathcal{O}( \pm 1) \mathrm{eV}^{2}$ between the relevant vacuum mass eigenstates greatly exceeds those for mixing among ac- 
tive neutrinos, and collective oscillations among active neutrinos are expected to be suppressed due to the high matter density in the region of interest to us 36]. We further assume that nearly all neutrinos freely stream through this region due to their large mean-free-path. Under these assumptions, a $\nu_{e}-\nu_{s}$ resonance occurs when

$$
\frac{\delta m^{2}}{2 E_{\nu}} \cos 2 \theta=\frac{3 \sqrt{2}}{2} G_{F} n_{b}\left(Y_{e}-\frac{1}{3}\right)=V_{\nu_{e}}^{\mathrm{eff}},
$$

where $\theta$ is the vacuum mixing angle, $E_{\nu}$ is the neutrino energy (with average values of $\left\langle E_{\nu}\right\rangle \sim 10-15 \mathrm{MeV}$ ), $G_{F}$ is the Fermi coupling constant, and $n_{b}=\rho / m_{u}$ is the baryon number density with $m_{u}$ being the atomic mass unit. The right-hand side of Eq. (2) is the effective potential $V_{\nu_{e}}^{\text {eff }}$ from $\nu_{e}$ forward scattering on neutrons, protons, and $e^{\nu_{e}}$ in matter 17,18$]$. We neglect the contribution of $\nu-\nu$ forward scattering 37 39], which is only $\sim 1 \%$ of $V_{\nu_{e}}^{\mathrm{eff}}$ around the IR region.

In supernovae, the central $Y_{e}$ is $\lesssim 0.3$ after core bounce as a result of electron capture on nuclei and free protons during core collapse [40, 41]. With increasing radius, $Y_{e}$ becomes as low as 0.1 near the neutrinospheres, due to electron capture on shock-dissociated material. At even larger radii, $Y_{e}$ increases to $\sim 0.5$ due to reactions (1a) and (1b) (see Fig. (1a). Consequently, above the $\nu_{e}$-sphere at $r=R_{\nu_{e}}$, there is a radius, $R_{\mathrm{IR}}$, where $Y_{e}=1 / 3^{+}$ but $\sqrt{2} G_{F} n_{b} \gg \delta m^{2} / 2 E_{\nu}$, and Eq. (2) is satisfied for $\delta m^{2}>0$ (normal hierarchy). For typical density profiles, this IR corresponds to $\rho \sim 10^{9}-10^{12} \mathrm{~g} \mathrm{~cm}^{-3}$. A second outer resonance $(\mathrm{OR})$ occurs further out at larger values of $Y_{e}$ once $n_{b}$ drops enough to satisfy Eq. (2) (see Fig.(1)). The condition for a $\bar{\nu}_{e}-\bar{\nu}_{s}$ resonance differs from Eq. (2) by an opposite sign of the effective potential:

$$
\frac{\delta m^{2}}{2 E_{\nu}} \cos 2 \theta=-\frac{3 \sqrt{2}}{2} G_{F} n_{b}\left(Y_{e}-\frac{1}{3}\right)=V_{\bar{\nu}_{e}}^{\text {eff }} .
$$

If $\delta m^{2}>0$, an IR occurs for $\bar{\nu}_{e} \bar{\nu}_{s}$ conversion for $Y_{e}=$ $1 / 3^{-}$but there is no OR in this case. If $\delta m^{2}<0$ (inverted hierarchy), there would be only an IR for $\nu_{e}-\nu_{s}$ conversion but both an IR and an OR for $\bar{\nu}_{e}-\bar{\nu}_{s}$ conversion.

It is clear from the above discussion that independent of the mass hierarchy and for $\left|\delta m^{2}\right| \lesssim 10 \mathrm{eV}^{2}$, there is always an IR for both $\nu_{e}-\nu_{s}$ and $\bar{\nu}_{e}-\bar{\nu}_{s}$ conversion at $Y_{e} \approx$ $1 / 3$ in supernovae. [There is only an OR for $\nu_{x}-\nu_{s}$ or $\bar{\nu}_{x^{-}}$ $\bar{\nu}_{s}(x=\mu, \tau)$ conversion as $\left.V_{\nu_{x}}^{\text {eff }} \propto\left(1-Y_{e}\right)\right]$. However, an inverted hierarchy appears disfavored by neutrino mass constraints from the Cosmic Microwave Background and Tritium decay experiments [42 44]. Therefore, we will not discuss this case further but will focus on the IR at $Y_{e} \approx 1 / 3$ for a normal hierarchy with $\delta m^{2}>0$ below.

As $\sqrt{2} G_{F} n_{b} \gg \delta m^{2} / 2 E_{\nu}$ in the region of interest, the local effective neutrino mass eigenstates are determined by $V_{\nu_{e}}^{\text {eff }}$ except near the IR. At $r<R_{\mathrm{IR}}$ where $Y_{e}<1 / 3$ gives $V_{\nu_{e}}^{\text {eff }}<0$, the lighter (heavier) effective mass eigenstate $\nu_{L}\left(\nu_{H}\right)$ is approximately $\nu_{e}\left(\nu_{s}\right)$, while at $r>R_{\mathrm{IR}}$
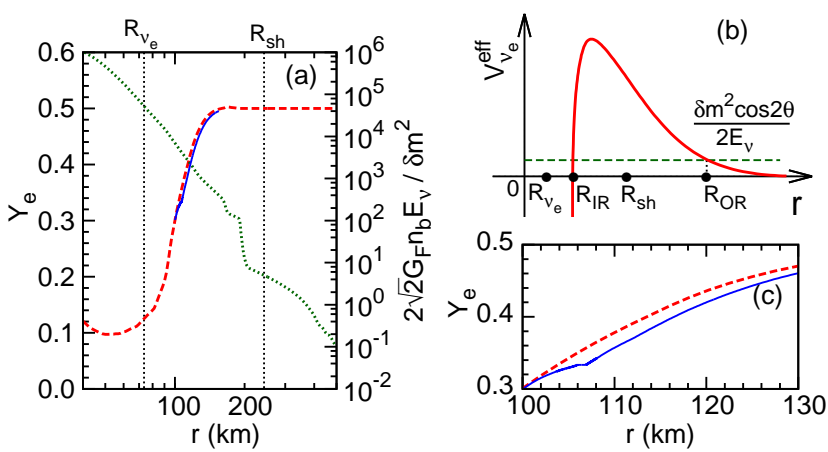

FIG. 1. (Color online) (a) $Y_{e}$ profile with (blue solid curve) and without (red dashed curve) ASFC for $\delta m^{2}=1.75 \mathrm{eV}^{2}$ and $\sin ^{2} 2 \theta=0.10$, and $2 \sqrt{2} G_{F} n_{b} E_{\nu} / \delta m^{2}$ for $E_{\nu}=10 \mathrm{MeV}$ (green dotted curve) at $t_{\mathrm{pb}} \sim 34 \mathrm{~ms}$. (b) Schematic plot of $V_{\nu_{e}}^{\text {eff }}$ (red solid curve) as a function of radius, illustrating the positions of different resonances with respect to the $\nu_{e}$-sphere $\left(R_{\nu_{e}}\right)$ and the shock $\left(R_{\mathrm{sh}}\right)$. (c) Blow-up of the $Y_{e}$ profiles in (a) for $r \gtrsim R_{\mathrm{IR}}$.

where $Y_{e}>1 / 3$ gives $V_{\nu_{e}}^{\text {eff }}>0, \nu_{L}\left(\nu_{H}\right)$ is approximately $\nu_{s}\left(\nu_{e}\right)$. With $V_{\overline{\nu_{e}}}^{\text {eff }}=-V_{\nu_{e}}^{\text {eff }}$, the situation is exactly the opposite for the effective antineutrino mass eigenstates. As $\nu_{e}\left(\bar{\nu}_{e}\right)$ cross $R_{\mathrm{IR}}$ from below, the probability that they hop from $\nu_{L}\left(\bar{\nu}_{H}\right)$ to $\nu_{H}\left(\bar{\nu}_{L}\right)$ can be approximated by the Landau-Zener formula [46, 47]:

$$
P_{\text {hop }}\left(E_{\nu}, \mu\right)=\exp \left(-\frac{\pi^{2}}{2} \frac{\delta r}{L}\right)
$$

where $\delta r=\delta m^{2} \sin 2 \theta /\left(\mu E_{\nu}\left|d V_{\nu_{e}\left(\bar{\nu}_{e}\right)}^{\text {eff }} / d r\right|_{\text {res }}\right)$ is the width of the resonance region with $\mu$ the cosine of the angle between the $\nu_{e}\left(\bar{\nu}_{e}\right)$ momentum and the radial direction, and $L=4 \pi E_{\nu} /\left(\delta m^{2} \sin 2 \theta\right)$ is the oscillation length at resonance [48]. Taking $\delta m^{2}=1.75 \mathrm{eV}^{2}$ and $\sin ^{2} 2 \theta=0.10$, which are the best-fit parameters inferred from reactor neutrino experiments 12], we obtain $L \approx 45 \mathrm{~m}$ for $E_{\nu}=10 \mathrm{MeV}$. As $\left|Y_{e}-1 / 3\right| \ll 1$ at the IR, $\left|d V_{\nu_{e}\left(\bar{\nu}_{e}\right)}^{\mathrm{eff}} / d r\right|_{\mathrm{res}} \approx(3 \sqrt{2} / 2) G_{F}\left(\rho_{\mathrm{res}} / m_{u}\right)\left|d Y_{e} / d r\right|_{\mathrm{res}}$. Using the same mixing parameters as above and taking $\rho_{\text {res }}=10^{9} \mathrm{~g} \mathrm{~cm}^{-3},\left|d Y_{e} / d r\right|_{\text {res }}=10^{-2} \mathrm{~km}^{-1}$, and $\mu=1$, we obtain $\delta r \approx 48 \mathrm{~m}$ for $E_{\nu}=10 \mathrm{MeV}$. In the above example, $\delta r \gtrsim L$ gives $P_{\text {hop }} \sim 0$, which means that $\nu_{e}$ $\left(\bar{\nu}_{e}\right)$ produced originally as $\nu_{L}\left(\bar{\nu}_{H}\right)$ will stay in their local effective mass eigenstates, and after crossing the IR, be completely converted into $\nu_{s}\left(\bar{\nu}_{s}\right)$.

In general, $\delta r / L \propto\left(\delta m^{2} \sin 2 \theta / E_{\nu}\right)^{2}\left(\rho\left|d Y_{e} / d r\right|\right)_{\text {res }}^{-1} / \mu$ and therefore, $P_{\text {hop }}\left(E_{\nu}, \mu\right)$ is sensitive to $d Y_{e} / d r$ at $Y_{e} \approx$ $1 / 3$. We define $E_{0.5}$ as the $E_{\nu}$ corresponding to $P_{\text {hop }}=$ 0.5 for $\mu=1$. It is clear from Eq. (4) and the above discussion that most of the $\nu_{e}\left(\bar{\nu}_{e}\right)$ with $E_{\nu}<E_{0.5}$ will be converted into $\nu_{s}\left(\bar{\nu}_{s}\right)$ after passing through the IR $\left(P_{\text {hop }} \sim 0\right)$, while most of those with $E_{\nu}>E_{0.5}$ will survive in their initial flavor states $\left(P_{\text {hop }} \sim 1\right)$.

The sensitivity of $P_{\text {hop }}$ to $d Y_{e} / d r$ at $Y_{e} \approx 1 / 3$ requires special attention. In the dynamic environment of a su- 
pernova, the $k$ th mass element is characterized by its radius $r_{k}(t)$, temperature $T_{k}(t)$, density $\rho_{k}(t)$, and electron fraction $Y_{e, k}(t)$ as functions of time $t$. The profile $Y_{e}(r, t)$ at a specific $t$ is obtained from the sets $\left\{Y_{e, k}(t)\right\}$ and $\left\{r_{k}(t)\right\}$ formed by all mass elements. The time evolution of $Y_{e, k}(t)$ is governed by

$$
\begin{aligned}
\frac{d Y_{e, k}}{d t}= & {\left[\lambda_{\nu_{e} n, k}(t)+\lambda_{e^{+} n, k}(t)\right] Y_{n, k}(t) } \\
& -\left[\lambda_{\bar{\nu}_{e} p, k}(t)+\lambda_{e^{-} p, k}(t)\right] Y_{p, k}(t),
\end{aligned}
$$

where $Y_{n, k}(t)$ and $Y_{p, k}(t)$ are the neutron and proton fraction, respectively, and $\lambda_{\alpha \beta, k}(t)$ corresponds to the rate per target nucleon for reactions (1a) and (1b) and their reverse reactions in the mass element. For $T_{k} \gtrsim 10^{10} \mathrm{~K}$, $Y_{n, k} \approx 1-Y_{e, k}$ and $Y_{p, k} \approx Y_{e, k}$. In general, $Y_{n, k}(t)$ and $Y_{p, k}(t)$ can be followed with a nucleosynthesis network given $T_{k}(t), \rho_{k}(t)$, and $Y_{e, k}(t)$, from which $\lambda_{e^{+}{ }_{n, k}}(t)$ and $\lambda_{e^{-} p, k}(t)$ can also be calculated. As $\lambda_{\nu_{e} n, k}(t)$ and $\lambda_{\bar{\nu}_{e} p, k}(t)$ used to determine $Y_{e, k}(t)$ are affected by $\nu_{e}-\nu_{s}$ and $\bar{\nu}_{e}-\bar{\nu}_{s}$ conversion, which in turn depends on $Y_{e}(r, t)$ obtained from the set $\left\{Y_{e, k}(t)\right\}$, we must treat this feedback in calculating $P_{\text {hop }}$.

We use the data from an $8.8 M_{\odot}$ electron-capture supernova (ECSN) simulation [45], which features the only successful explosion in spherical symmetry with threeflavor Boltzmann neutrino transport [45, 49]. In this model, an early onset of the explosion occurs at time post core bounce $t_{\mathrm{pb}} \sim 38 \mathrm{~ms}$, in qualitative agreement with multi-dimensional simulations [50]. We take the sets $\left\{r_{k}(t)\right\},\left\{T_{k}(t)\right\}$, and $\left\{\rho_{k}(t)\right\}$ from the simulation and increase the resolution by adding $\sim 2,000$ mass elements in the IR region so that the resonance can be resolved properly. We recalculate each $Y_{e, k}(t)$ using Eq. (5) to obtain the self-consistent $Y_{e}(r, t)$ in the presence of ASFC. As initial values of $Y_{e, k}(t)$, we use the $Y_{e}$ profile of the simulation at $t_{\mathrm{pb}} \approx 30 \mathrm{~ms}$ when the shock has already passed through the IR region. Subsequently, we use the recalculated high-resolution $Y_{e}$ profile to determine which $\nu_{e}\left(\bar{\nu}_{e}\right)$ have crossed the IR and compute their hopping probabilities, $P_{\text {hop }}\left(E_{\nu}, \mu\right)$. These probabilities are then multiplied by the distribution function of the $\nu_{e}\left(\bar{\nu}_{e}\right), f_{\nu_{e}\left(\bar{\nu}_{e}\right)}\left(E_{\nu}, \mu\right)$, given by the simulation to determine $\lambda_{\nu_{e} n, k}\left(\lambda_{\bar{\nu}_{e} p, k}\right)$ in Eq. (5).

We first consider the results for $\delta m^{2}=1.75 \mathrm{eV}^{2}$ and $\sin ^{2} 2 \theta=0.10$. We compare the original $Y_{e}$ profile at $t_{\mathrm{pb}} \sim 34 \mathrm{~ms}$ with the one obtained by including ASFC feedback in Fig. 17. ASFC results in lower $Y_{e}$ values and produces a plateau at $Y_{e}=1 / 3^{+}$corresponding to $r \sim 106 \mathrm{~km}$. The time evolution of $R_{\nu_{e}}$, shock radius $R_{\mathrm{sh}}$, and $R_{\mathrm{IR}}$ and $E_{0.5}$ with and without ASFC feedback is shown in Fig. 2 If ASFC feedback is neglected, all $\nu_{e}$ and $\bar{\nu}_{e}$ of different $E_{\nu}$ have approximately the same $R_{\mathrm{IR}}$. In this case, $E_{0.5}$ and $R_{\mathrm{IR}}$ follow a similar trend: they increase due to the flattening of the $Y_{e}$ profile during the initial shock expansion, and decrease later due to the steepening of the $Y_{e}$ profile during the protoneutron
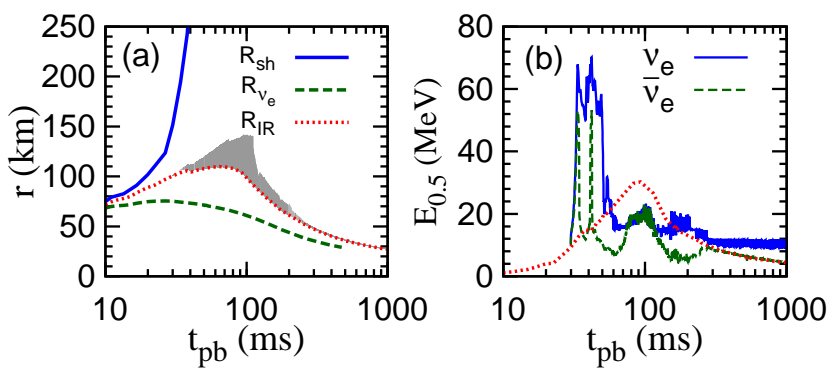

FIG. 2. (Color online) (a) Evolution of $R_{\nu_{e}}$ (green dashed curve), $R_{\mathrm{sh}}$ (blue solid curve), and $R_{\mathrm{IR}}$ for the original (red dotted curve) and recalculated (shaded region) $Y_{e}$ profiles. (b) Evolution of $E_{0.5}$ for the original (red dotted curve) and recalculated (blue solid and green dashed curves) $Y_{e}$ profiles. All results for ASFC assume $\delta m^{2}=1.75 \mathrm{eV}^{2}$ and $\sin ^{2} 2 \theta=$ 0.10 .

star cooling. Inclusion of ASFC feedback significantly extends the IR region in radius for times, $t_{\mathrm{pb}} \sim 32-$ $200 \mathrm{~ms}$, due to the formation of a plateau at $Y_{e}=1 / 3^{+}$. The $Y_{e}$ plateau greatly reduces $\left|d Y_{e} / d r\right|$ in the IR region and affects $\nu_{e}$ more than $\bar{\nu}_{e}$ as the latter cross the IR at $Y_{e}=1 / 3^{-}$. Consequently, $E_{0.5}$ differs for $\nu_{e}$ and $\bar{\nu}_{e}$ with $E_{0.5, \nu_{e}} \gtrsim E_{0.5, \bar{\nu}_{e}}$. Compared to the case without ASFC feedback, both $E_{0.5, \nu_{e}}$ and $E_{0.5, \bar{\nu}_{e}}$ rise much faster to larger values greatly exceeding $\left\langle E_{\nu}\right\rangle$ during the initial shock expansion, i.e., most of $\nu_{e}$ and $\bar{\nu}_{e}$ are converted into sterile counterparts. At later times, $E_{0.5, \bar{\nu}_{e}}$ decreases while $E_{0.5, \nu_{e}}$ remains at $\sim 10 \mathrm{MeV} \sim\left\langle E_{\nu_{e}}\right\rangle$.

The effect of the plateau at $Y_{e}=1 / 3^{+}$on $E_{0.5, \nu_{e}}$ enhances conversion of $\nu_{e}$ into $\nu_{s}$, which greatly decreases $Y_{e}$ at larger radii by reducing the rate of reaction (1a). We show the $Y_{e}$ evolution for an example mass element as a function of time in Fig. 3a with and without ASFC feedback. As the mass element is being ejected, it encounters the plateau of $Y_{e}=1 / 3^{+}$at $t_{\mathrm{pb}} \sim 90-120 \mathrm{~ms}$ and its $Y_{e}$ is greatly reduced from the original supernova simulation values (from 0.49 to $0.37-0.39$ for $t_{\mathrm{pb}} \gtrsim 200 \mathrm{~ms}$ ). This reduction is mainly driven by $e^{-}$capture on protons, the inverse of reaction (1a), and by absorption on protons of the surviving $\bar{\nu}_{e}$, reaction (1b), after most $\nu_{e}$ have been converted into $\nu_{s}$.

Similar reduction of $Y_{e}$ by ASFC occurs in $\sim 10^{-2} M_{\odot}$ of ejecta. The integrated nucleosynthesis in this material is shown in Fig. 3b. Compared to the case without ASFC where only elements with $Z \lesssim 30$ are produced, much heavier elements from $Z=38$ (Sr) to $Z=48$ $(\mathrm{Cd})$ are produced with ASFC and their pattern is in broad agreement with observations of the metal-poor star HD 122563 [51, 52]. It remains to be explored if ASFC can help to overcome the difficulties of neutrino-driven winds from more massive supernovae in producing elements with $Z>42$ [53]. ECSN differs from those models by the presence of a dynamically ejected neutron-rich 

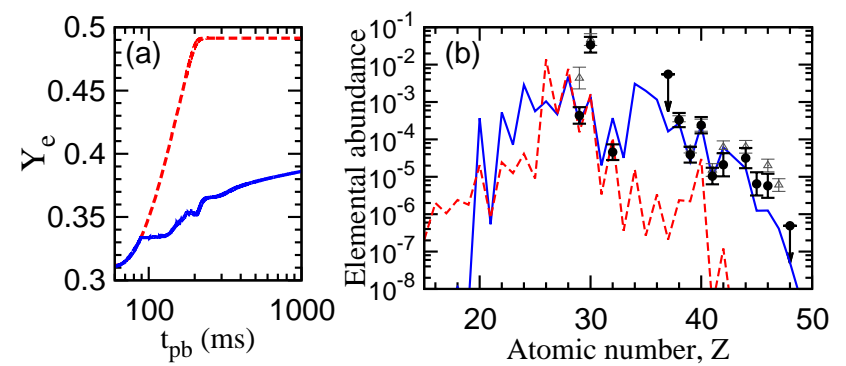

FIG. 3. (Color online) Comparison of (a) $Y_{e}$ evolution in an example mass element and (b) integrated nucleosynthesis with (blue solid curve) and without (red dashed curve) ASFC. Data on the metal-poor star HD 122563 normalized to the calculated abundance of $\operatorname{Zr}(Z=40)$ with ASFC are shown as open triangles [51] and filled circles [52] in (b).

component 54]. This material is ejected at $t_{\mathrm{pb}} \lesssim 100 \mathrm{~ms}$ and its $Y_{e}$ is reduced to $\sim 0.38$ by ASFC, thereby enabling production of elements with $Z>42$.

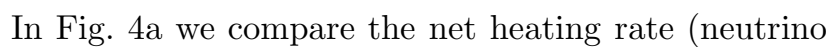
heating minus matter cooling) with and without ASFC as a function of radius at $t_{\mathrm{pb}} \sim 34 \mathrm{~ms}$. It can be seen that ASFC drastically turns the region of net heating at $r \gtrsim 110 \mathrm{~km}$ into one of net cooling. So far we have focused on the results for $\delta m^{2}=1.75 \mathrm{eV}^{2}$ and $\sin ^{2} 2 \theta=0.10$. In view of the potential implications of ASFC for supernova explosion, we examine a wide range of mixing parameters. In Fig. 4 $4 \mathrm{~b}$ we show contours of $\dot{q}_{\nu_{e} n}^{\prime} / \dot{q}_{\nu_{e} n}$ and $\dot{q}_{\bar{\nu}_{e} p}^{\prime} / \dot{q}_{\bar{\nu}_{e} p}$ for $r>R_{\mathrm{IR}}$ at $t_{\mathrm{pb}} \sim 34 \mathrm{~ms}$ in the $\left(\sin ^{2} 2 \theta, \delta m^{2}\right)$ space, where $\dot{q}_{\nu_{e} n}^{\prime}$ and $\dot{q}_{\bar{\nu}_{e} p}^{\prime}$ are the heating rates for reactions (1a) and (1b) with ASFC, respectively, and the unprimed counterparts are for the case without ASFC. The filled diamond in Fig. 4b represents the mixing parameters used above and the shaded regions give those inferred from reactor neutrino experiments at the $90 \%$ confidence level [12]. Except for the two regions with the lowest $\delta m^{2}$, all other inferred parameters for ASFC might have a large negative impact on the explosion of the $8.8 M_{\odot}$ ECSN.

We have shown that the existence of sterile neutrinos with parameters inferred from reactor neutrino experiments produces substantial ASFC of the $\nu_{e}-\nu_{s}$ and $\bar{\nu}_{e}-\bar{\nu}_{s}$ types near the core of an $8.8 M_{\odot}$ ECSN. As a result of ASFC feedback, a $Y_{e}$ plateau is formed in the resonance region where $Y_{e} \approx 1 / 3$. This further enhances conversion of $\nu_{e}$ into $\nu_{s}$, thereby reducing $Y_{e}$ at larger radii. For the inferred best-fit parameters, nuclei with $Z>40$ are produced in a total $\sim 10^{-2} M_{\odot}$ of supernova ejecta with a pattern in broad agreement with metal-poor star observations. Without ASFC, only nuclei with $Z \lesssim 30$ are produced. However, for a wide range of mixing parameters, the neutrino heating rates are strongly suppressed by ASFC at times when such heating is important for energizing the shock. A caveat of our treatment is that sup-
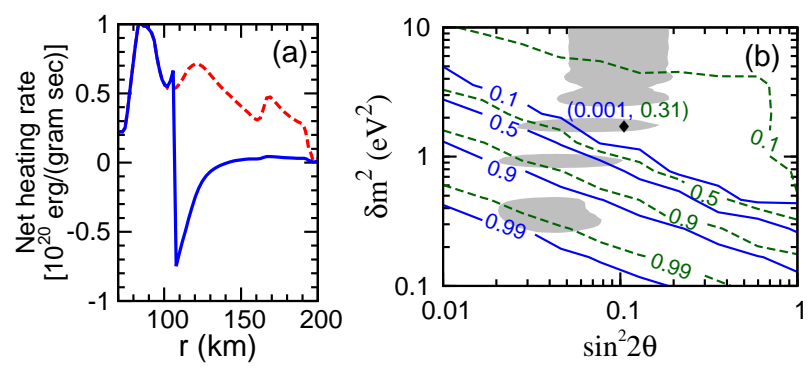

FIG. 4. (Color online) (a) Comparison of the net heating rate with (blue solid curve) and without (red dashed curve) ASFC as a function of radius at $t_{\mathrm{pb}} \sim 34 \mathrm{~ms}$ for $\delta m^{2}=1.75 \mathrm{eV}^{2}$ and $\sin ^{2} 2 \theta=0.10$ [filled diamond in (b)]. (b) Contours of $\dot{q}_{\nu_{e} n}^{\prime} / \dot{q}_{\nu_{e} n}$ (blue solid curves) and $\dot{q}_{\bar{\nu}_{e} p}^{\prime} / \dot{q}_{\bar{\nu}_{e} p}$ (green dashed curves) for $r>R_{\mathrm{IR}}$ at $t_{\mathrm{pb}} \sim 34 \mathrm{~ms}$. Numbers in parentheses give $\left(\dot{q}_{\nu_{e} n}^{\prime} / \dot{q}_{\nu_{e} n}, \dot{q}_{\bar{\nu}_{e} p}^{\prime} / \dot{q}_{\bar{\nu}_{e} p}\right)$ for the mixing parameters used in (a). Shaded regions give mixing parameters inferred at the $90 \%$ confidence level by [12].

pression of neutrino heating by ASFC would likely change the dynamic and thermodynamic conditions. Thus, the exact effects of ASFC on supernova explosion and nucleosynthesis remain to be studied by implementing ASFC in the simulations self-consistently. These studies should also be extended to supernova models for more massive progenitors. Our results suggest that such studies can strongly constrain the mixing parameters for ASFC in combination with neutrino experiments and cosmological considerations. In the future we will examine the effects of ASFC in supernovae along with other flavor conversion processes and determine the impact on neutrino signals in terrestrial detectors. These studies along with selfconsistent treatment of neutrino flavor transformation in supernovae can not only provide unique probes of neutrino mixing, but may also help understanding supernova explosion and nucleosynthesis.

M.-R.W. is supported by the Alexander von Humboldt Foundation. T.F. acknowledges support from the Narodowe Centrum Nauki (NCN) within the "Maestro" program under contract No. DEC-2011/02/A/ST2/00306. L.H. and G.M.P. are partly supported by the Deutsche Forschungsgemeinschaft through contract SFB 634, the Helmholtz International Center for FAIR within the framework of the LOEWE program launched by the state of Hesse and the Helmholtz Association through the Nuclear Astrophysics Virtual Institute (VH-VI-417). Y.Z.Q. is partly supported by the US DOE (DE-FG0287ER40328). We gratefully thank Hans-Thomas Janka, Irene Tamborra, and two anonymous reviewers for helpful comments and suggestions.

[1] S. Schael et al. (ALEPH Collaboration, DELPHI Collaboration, L3 Collaboration, OPAL Collaboration, 
SLD Collaboration, LEP Electroweak Working Group, SLD Electroweak Group, SLD Heavy Flavour Group), Phys.Rept. 427, 257 (2006).

[2] J. Beringer et al. (Particle Data Group), Phys.Rev. D86, 010001 (2012)

[3] A. Aguilar-Arevalo et al. (LSND Collaboration), Phys.Rev. D64, 112007 (2001)

[4] A. Aguilar-Arevalo et al. (MiniBooNE Collaboration), Phys.Rev.Lett. 105, 181801 (2010).

[5] A. Aguilar-Arevalo et al. (MiniBooNE Collaboration), (2013), arXiv:1303.2588 [hep-ex]

[6] G. Mention, M. Fechner, T. Lasserre, T. Mueller, D. Lhuillier, et al., Phys.Rev. D83, 073006 (2011)

[7] M. A. Acero, C. Giunti, and M. Laveder, Phys.Rev. D78, 073009 (2008)

[8] K. Abazajian, M. Acero, S. Agarwalla, A. Aguilar-Arevalo, C. Albright, et al., (2012), arXiv:1204.5379 [hep-ph]

[9] M. Archidiacono, N. Fornengo, C. Giunti, S. Hannestad, and A. Melchiorri, (2013), arXiv:1302.6720 [astro-ph.CO].

[10] J. Hamann, S. Hannestad, G. G. Raffelt, I. Tamborra, and Y. Y. Wong, Phys.Rev.Lett. 105, 181301 (2010)

[11] G. Mangano and P. D. Serpico, Phys.Lett. B701, 296 (2011)

[12] J. Kopp, P. A. N. Machado, M. Maltoni, and T. Schwetz, (2013), arXiv:1303.3011 [hep-ph]

[13] C. Giunti, M. Laveder, Y. Li, and H. Long, Phys.Rev. D88, 073008 (2013) arXiv:1308.5288 [hep-ph]

[14] A. Mirizzi, G. Mangano, N. Saviano, E. Borriello, C. Giunti, et al., (2013), arXiv:1303.5368 [astro-ph.CO]

[15] L. Wolfenstein, Phys. Rev. D17, 2369 (1978).

[16] S. P. Mikheyev and A. Y. Smirnov, Sov. J. Nucl. Phys. 42, 913 (1985).

[17] K. Kainulainen, J. Maalampi, and J. Peltoniemi, Nucl.Phys. B358, 435 (1991).

[18] H. Nunokawa, J. Peltoniemi, A. Rossi, and J. Valle, Phys.Rev. D56, 1704 (1997)

[19] X. Shi and G. Sigl, Phys.Lett. B323, 360 (1994)

[20] S. Pastor, V. Semikoz, and J. Valle, Astropart.Phys. 3, 87 (1995)

[21] D. O. Caldwell, G. M. Fuller, and Y.-Z. Qian, Phys.Rev. D61, 123005 (2000)

[22] J. Fetter, G. McLaughlin, A. Balantekin, and G. Fuller, Astropart.Phys. 18, 433 (2003)

[23] J. Beun, G. McLaughlin, R. Surman, and W. Hix, Phys.Rev. D73, 093007 (2006)

[24] J. Hidaka and G. M. Fuller, Phys.Rev. D74, 125015 (2006)

[25] S. Choubey, N. Harries, Phys.Rev. D76, 073013 (2007)

[26] J. Hidaka and G. M. Fuller,
Phys.Rev. D76, 083516 (2007)

[27] G. M. Fuller, A. Kusenko, and K. Petraki, Phys.Lett. B670, 281 (2009).

[28] I. Tamborra, G. G. Raffelt, L. Hudepohl, and H.-T. Janka, JCAP 1201, 013 (2012).

[29] H. A. Bethe and R. Wilson, James, Astrophys.J. 295, 14 (1985)

[30] B. Müller, H.-T. Janka, and A. Marek, Astrophys.J. 756, 84 (2012)

[31] Y. Suwa, T. Takiwaki, K. Kotake, T. Fischer, M. Liebendrfer, et al., Astrophys.J. 764, 99 (2013).

[32] S. W. Bruenn, A. Mezzacappa, W. R. Hix, E. J. Lentz, O. B. Messer, et al., Astrophys.J. 767, L6 (2013).

[33] Y.-Z. Qian, G. M. Fuller, G. J. Mathews, R. Mayle, J. R. Wilson, et al., Phys.Rev.Lett. 71, 1965 (1993).

[34] Y. Qian and S. Woosley, Astrophys.J. 471, 331 (1996).

[35] A. Arcones and F.-K. Thielemann, J.Phys. G40, 013201 (2013)

[36] A. Esteban-Pretel, A. Mirizzi, S. Pastor, R. Tomas, G. Raffelt, et al., Phys.Rev. D78, 085012 (2008)

[37] G. Sigl and G. Raffelt, Nucl.Phys. B406, 423 (1993).

[38] G. M. Fuller, R. W. Mayle, J. R. Wilson, and D. N. Schramm, Astrophys. J. 322, 795 (1987)

[39] J. T. Pantaleone, Phys.Lett. B287, 128 (1992)

[40] W. Hix, O. Messer, A. Mezzacappa, M. Liebendoerfer, J. Sampaio, et al., Phys.Rev.Lett. 91, 201102 (2003)

[41] K. Langanke, G. Martinez-Pinedo, J. Sampaio, D. Dean, W. Hix, et al., Phys.Rev.Lett. 90, 241102 (2003).

[42] G. Hinshaw, D. Larson, E. Komatsu, D. Spergel, C. Bennett, et al., (2012), arXiv:1212.5226 [astro-ph.CO].

[43] P. Ade et al. (Planck Collaboration), (2013), arXiv:1303.5076 [astro-ph.CO]

[44] C. Kraus, B. Bornschein, L. Bornschein, J. Bonn, B. Flatt, et al., Eur.Phys.J. C40, 447 (2005).

[45] T. Fischer, S. Whitehouse, A. Mezzacappa, F.-K. Thielemann, and M. Liebendorfer, Astron.Astrophys. 517, A80 (2010)

[46] L. Landau, Phys. Z. Sowjetunion 2, 46 (1932).

[47] C. Zener, Proc.Roy.Soc.Lond. A137, 696 (1932).

[48] W. Haxton, R. Hamish Robertson, and A. M. Serenelli, (2012), arXiv:1208.5723 [astro-ph.SR]

[49] L. Hudepohl, B. Muller, H.-T. Janka, A. Marek, and G. Raffelt, Phys.Rev.Lett. 104, 251101 (2010).

[50] H.-T. Janka, B. Mueller, F. Kitaura, and R. Buras, Astron.Astrophys. 485, 199 (2007).

[51] S. Honda, W. Aoki, Y. Ishimaru, S. Wanajo, and S. Ryan, Astrophys.J. 643, 1180 (2006)

[52] I. U. Roederer, J. E. Lawler, J. S. Sobeck, T. C. Beers, J. J. Cowan, et al., Astrophys.J.Suppl. 203, 27 (2012)

[53] G. Martnez-Pinedo, T. Fischer, and L. Huther, (2013), arXiv:1309.5477 [astro-ph.HE]

[54] S. Wanajo, H.-T. Janka, and B. Mueller, Astrophys.J. 726, L15 (2010) 